\title{
The effect of autologous platelet rich plasma on tenocytes of the human rotator cuff
}

\author{
Stephan Pauly ${ }^{1 * \dagger} \mathbb{D}$, Franka Klatte-Schulz ${ }^{1,2 \dagger}$, Katharina Stahnke ${ }^{1}$, Markus Scheibel ${ }^{1}$ and Britt Wildemann $n^{1,2,3}$
}

\begin{abstract}
Background: Platelet rich plasma (PRP) is widely used in rotator cuff repairs but its effect on the healing process is unclear. Several cell culture studies on the effect of allogenic PRP have reported promising results but are not transferable to clinical practice.

The aim of the present study is to assess the possible effect of autologous PRP on rotator cuff tendon cells. The amount of growth factors involved with tendon-bone healing (PDGF-AB, IGF-1, TGF- $\beta 1$, BMP-7 and -12) is quantified.
\end{abstract}

Methods: Rotator cuff tissue samples were obtained from $(n=24)$ patients grouped by age $(>/<65$ years) and sex into four groups and cells were isolated and characterized. Later, autologous PRP preparations were obtained and the effect was analyzed by means of cell proliferation, collagen I synthesis and expression of collagen I and III. Furthermore, the PRPs were quantified for growth factor content by means of platelet-derived growth factor (PDGF-AB), insulin-like growth factor (IGF-1), transforming growth factor (TGF- $\beta 1$ ), as well as bone morphogenetic protein (BMP) -7 and - 12 .

Results: Cell proliferation and absolute synthesis of collagen I were positively affected by PRP exposure compared to controls $(p<0.05)$, but expression and relative synthesis of collagen I (normalized to cell proliferation) were significantly reduced.

PRP contained high amounts of IGF-1 and lower levels of TGF- $\beta 1$ and PDGF-AB. The amounts of BMP-7 and -12 were below the detection limits.

Conclusions: PRP is a source of growth factors such involved with tendon-bone healing. PRP had an anabolic effect on the human rotator cuff tenocytes of the same individual in vitro by means of cell proliferation and absolute, but not relative collagen I synthesis.

These results encourage further studies on clinical outcomes with more comparable standards in terms of preparation and application methods.

Level of evidence: Controlled laboratory study.

Keywords: PRP, Platelet rich, Rotator cuff, Tenocyte, Tendon cell, Autologous, Growth factor

\section{Background}

The ongoing development of new rotator cuff (RC) repair techniques, such as double row repairs, has led to increased initial biomechanical stability, but recent meta-analyses have revealed that there is no clear clinical and radiographic advantage in terms of improved

\footnotetext{
* Correspondence: stephan.pauly@charite.de

tStephan Pauly and Franka Klatte-Schulz contributed equally to this work. ${ }^{1}$ Julius Wolff Institut, Center for Musculoskeletal Surgery,

Charité-Universitaetsmedizin, Augustenburger Platz 1, 13353 Berlin, Germany Full list of author information is available at the end of the article
}

tendon integration $[9,15,39,42,43,48,50]$. As non-healing still represents the main challenge following surgical rotator cuff repair, biological approaches have been suggested as a way to improve tendon-bone healing $[20,23,29,36]$. The use of growth factors seems to improve biological regeneration, but many of these are recombinant in origin and are often cost intensive to acquire [31].

Platelet rich plasma (PRP) as a supraphysiological concentration of platelets is an easily producible, low-cost source of several autologous growth factors [33]. 
However, available studies on the effect of PRP on clinical and radiographic parameters following RC repair do not just differ in their preparation and application methods, but also in their results. Recent meta-analyses have described either no clinical benefit or no consistent effect on re-tear rates, or at least uncertainty about the evidence $[5,7,30,47,51,58,60]$.

In-vitro studies on the effect of PRP have, however, described improved tendon matrix production and gene expression, but only PRP preparations that are allogenic in origin were applied to tenocyte cultures, mainly with no dedicated characterization, and these were primarily obtained from monadic numbers of donors [12, 13, 17, $22,24,46]$. In contrast, the process of assessing tendon cell cultures with autologous PRP from the same patient matches with the clinical application, but has not been described in the literature. This approach requires a protocol to first harvest and culture cells, followed by subsequent PRP production and application. Tendon cell characterization should also be part of the approach to verify the targeted cell type.

The presence of several growth factors involved with musculoskeletal repair, such as platelet-derived growth factor (PDGF), transforming growth factor- $\beta$ (TGF- $\beta$ ), insulin-like growth factor (IGF), epidermal growth factor (EGF), vascular endothelial growth factor (VEGF), and fibroblast growth factor (FGF), has been described within PRP preparations $[25,33,36,52]$. A focus on tenocyte metabolism and improved tendon-bone healing has highlighted further growth factors, such as bone morphogenetic protein 7 (BMP-7) [26, 27, 40, 56, 62] and 12 (BMP-12) [29, 49,59]. However, it is currently unknown whether these are present in PRP.

The present study aims to characterize a human PRP-preparation in terms of the quantity of various growth factors. In addition, it investigates the effect of autologous PRP on tenocyte-like cells for the first time.

We hypothesize that there will be improved cell proliferation and collagen I synthesis under stimulation with PRP.

\section{Methods}

\section{Study design, inclusion and exclusion criteria}

After receiving institutional review board approval, supraspinatus (SSP) tendon samples were taken intraoperatively from $n=24$ patients undergoing arthroscopic RC repair. Participation in the study did not affect the intra- and postoperative treatment strategy.

$(N=12)$ male and $(n=12)$ female patients with full thickness tears of the SSP tendon were included in the study. The exclusion criteria were partial- or massive tears (larger than Bateman $\left.\mathrm{III}^{\circ}[2]\right)$, tears with fatty infiltration greater than Goutallier $\mathrm{III}^{\circ}$ [18], and medical conditions precluding arthroscopic cuff repair.
Donor demographics, tendon material, surgery $(t=0)$ $N=12$ female ( $\varnothing$ age 64.5 years; range $50-74$ years) and $n=12$ male patients ( $\varnothing$ age 62.4 years; range, $38-$ 73 years) were included in the study and subdivided into four groups by age $(>/<65$ years $)$ and sex. The average age of all the patients at the time of surgery was 63.5 years.

\section{Cell isolation and culture}

During arthroscopic RC repair and after debridement with a shaver, all tendon biopsies were obtained 3-5 $\mathrm{mm}$ from the torn proximal tendon edge. Human tenocyte-like cells (hTLCs) were isolated from these SSP tendon biopsies by digestion with $0.3 \%$ collagenase type CLS II in PBS with $\mathrm{Ca}^{2+} / \mathrm{Mg}^{2+}$ for $2 \mathrm{~h}$ at $37^{\circ} \mathrm{C}$, as described previously [41]. The cells were cultured with a normal growth medium (DMEM/Ham's F12 with 10\% fetal calf serum (FCS), and 1\% Penicillin/Streptomycin, all Biochrom AG, Berlin, Germany) at $37^{\circ} \mathrm{C}$, with $95 \%$ humidity and $5 \%$ carbon dioxide, and with a change of medium every 2 to 3 days. The cells were cryo-preserved until they were used for the stimulation when they reached a minimum of $5 \times 10^{5}$ vital cells.

\section{Exemplary hTLC characterization}

A total of 8 of the 24 hTLC cultures were characterized according to previously published research [41]. The cells were analyzed for their expression of the tendon markers collagen I, scleraxis, and Mohawk homeobox, and the negative markers collagen II and osteocalcin. The relative gene expression levels were normalized to glyceraldehyde 3-phosphate dehydrogenase (GAPDH) and calculated using the $2^{-\Delta \mathrm{Ct}}$ method.

\section{PRP preparation}

Approximately 2.5 years after their surgery, the 12 male and 12 female SSP tendon patients were invited to donate blood for the PRP preparation. The fresh PRP was then used for the autologous stimulation of the hTLCs, which had previously been isolated from their torn SSP biopsies and stored until used for stimulation experiments. A commercially available kit was used for the standardized PRP preparation according to the manufacturer's instructions: Arthrex ACP ${ }^{\bullet}$ (Arthrex, Naples, FL), using $15 \mathrm{~mL}$ Arthrex $\mathrm{ACP}^{\circ}$ double syringes. An average of $5 \mathrm{~mL}$ of PRP was obtained from each of them. No additional anticoagulants were used. Hence, quick preparation and processing of the PRP were mandatory.

Directly after preparation, the PRP was analyzed for the platelet count using a haemocytometer (C-Chip, NanoEnTek, Seoul, Korea). After preparation, the PRP was used to stimulate the hTLCs, and was then subsequently cryo-preserved at $-20^{\circ} \mathrm{C}$ until the growth factor quantification. 


\section{PRP-stimulation}

A total of $1 \times 10^{4}$ vital cells per well were seeded in a 24-well plate in triplicate and cultured in a normal growth medium. One day after seeding, an alamarBlue assay (Biozym, Germany) was used to analyze the cell proliferation. The cells then received either a growth medium with 2\% FCS (negative control), a growth medium with 10\% FCS (positive control), or 10\% PRP in a growth medium with $2 \%$ FCS. Six wells were used to obtain enough RNA for the negative control. The PRP was applied to the cells in a polycarbonate cell culture insert with a pore size of $0.4 \mu \mathrm{m}$ (Nunc), which was placed on the cell layer. The experimental set up is shown in Fig. 1. After incubating the cells for 5 days at $37^{\circ} \mathrm{C}$, the cell proliferation was analyzed, the cell culture supernatant was collected for the analysis of the collagen I protein synthesis, and the RNA was isolated to analyze the gene expression.

\section{Analysis of cell proliferation}

An alamarBlue assay was used to indirectly measure cell proliferation by using the reducing power of viable cells. The medium was removed from the cells and $700 \mu \mathrm{l}$ of a $10 \%$ alamarBlue solution in a medium with $10 \%$ FCS was applied to the cells and they were then incubated for $3 \mathrm{~h}$ at $37^{\circ} \mathrm{C}$. Subsequently, each well was analyzed in duplicate using spectrophotometry at 570 $\mathrm{nm}$ versus $595 \mathrm{~nm}$ against the blank (10\% alamarBlue solution incubated without cells).

\section{Analysis of the collagen I protein synthesis}

The collagen I protein synthesis was measured from day-5 cryo-preserved cell culture supernatants after PRP stimulation with the MicroVue C1CP EIA kit
(TECOmedical, Germany). The collagen I protein synthesis was normalized to the cell proliferation on day 5 .

\section{Analysis of gene expression}

RNA was isolated from the cells on day 5 directly in the well using the NucleoSpin RNA II Kit (Machary Nagel, Germany) according to the manufacturer's instructions. Cells in triplicate/ 6 repeats (negative control) from each donor were pooled. RNA quantity and purity were analyzed with a Nanodrop ND-1000 spectrophotometer (PeqLab Biotechnologie, Germany). A total of $100 \mathrm{ng}$ of RNA was transcribed into complementary DNA (cDNA) using the qScript cDNA Supermix (Quanta BioSciences, USA) with the Epgradient Mastercycler (Eppendorf, Germany). For the quantitative real-time PCR (qRTPCR) analysis, $1.25 \mathrm{ng}$ of cDNA was used as the PCR template. A total of $20 \mu \mathrm{l}$ of Sybr Green mastermix was used for each well and consisted of: $12.5 \mathrm{ml}$ of Sybr Green Supermix (Quanta BioSciences), $6.5 \mu \mathrm{l}$ of RNase/ DNase-free water, and $1 \mu \mathrm{l}$ of a primer mix $(10 \mu \mathrm{M})$. All the primer sequences were designed using the Primer 3 software (Freeware; http://frodo.wi.mit.edu/primer3), and were produced by Tib Molbiol, Berlin, Germany (Table 1). A qRT-PCR program with an initial denaturation step for $3 \mathrm{~min}$ at $94^{\circ} \mathrm{C}$ was used, followed by an amplification program with 40 repeated cycles $\left(95^{\circ} \mathrm{C}\right.$ for $15 \mathrm{~s}$, amplification temperature for $45 \mathrm{~s}, 72^{\circ} \mathrm{C}$ for $30 \mathrm{~s}$ ), and a melting curve program. The results were analyzed with the Realplex Software (Eppendorf). The relative gene expression levels were normalized to GAPDH and calculated using the $2^{-\Delta C t}$ method.

\section{Growth factor quantification from the PRP}

Growth factors such as PDGF-AB, IGF-I, TGF- $\beta 1$, BMP-7, and BMP-12 (also known as growth and

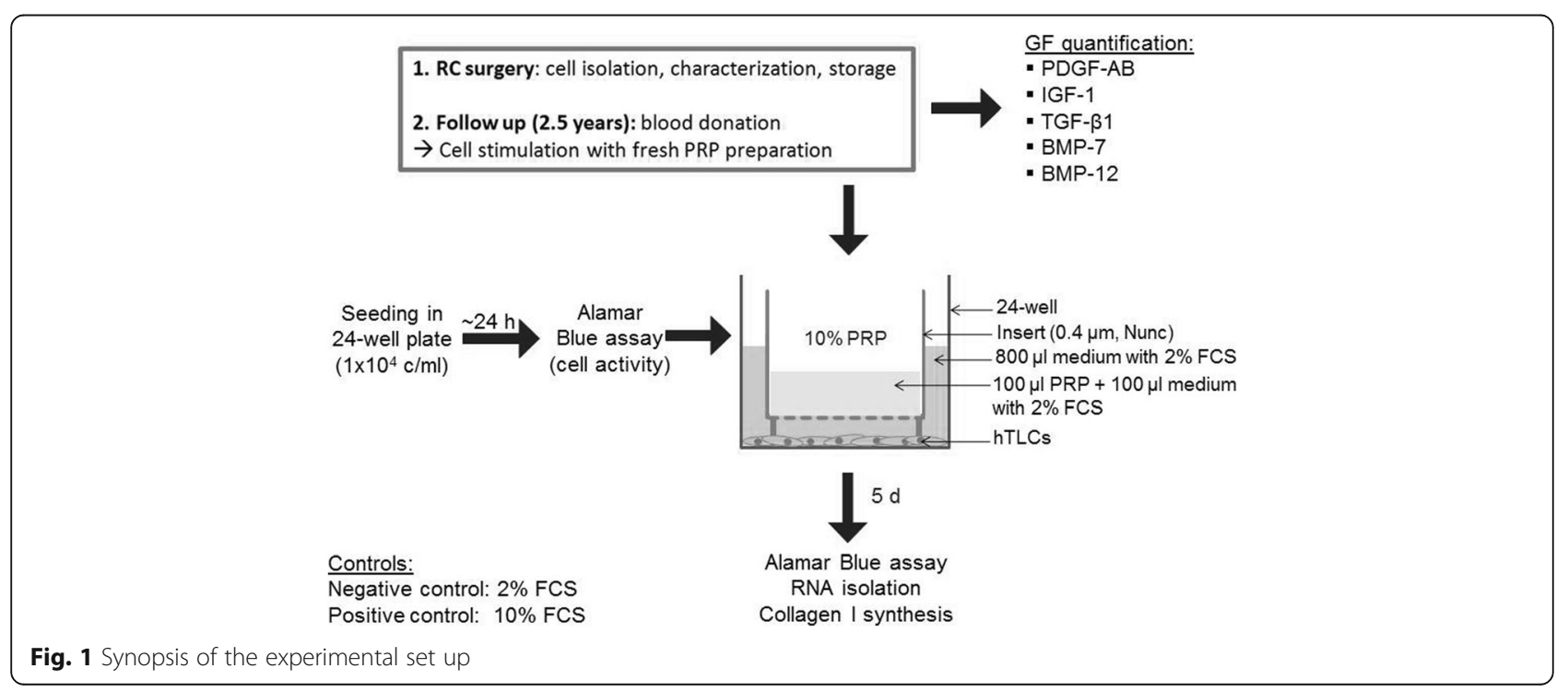


differentiation factor-7, GDF-7) were analyzed using ELISA according to the manufacturer's instructions. The PRP was thawed and clotted for about $30 \mathrm{~min}$ at room temperature and centrifuged at $1000 \times$ g for 10 min before being used for the ELISA analysis. The details for the ELISA kits are set out in Table 2. The growth factor concentration was normalized to the total protein content of the PRP, which was analyzed by a Coomassie Plus Protein Assay (Thermo Fisher Scientific, Germany).

\section{Statistics}

A statistical analysis was performed for 4 individual groups $(n=6)$ using the Mann-Whitney-U Test. The $P$-value was set to $p \leq 0.05$ and adjusted using the Bonferroni Holm correction. A Spearman's Rho correlation $\left(r_{s}\right)$ was used to analyze the correlation of different parameters.

\section{Results}

\section{Exemplary hTLC characterization}

The exemplary characterization of 8 hTLC cultures revealed high amounts of collagen I $\left(2^{-\Delta \mathrm{Ct}}=2.38 \pm 0.85\right)$ and an abundance of the tendon markers scleraxis $\left(2^{-\Delta \mathrm{Ct}}=0.003 \pm\right.$ $0.002)$ and Mohawk homeobox $\left(2^{-\Delta \mathrm{Ct}}=0.015 \pm 0.006\right)$ in accordance with previous studies [41]. Osteocalcin expression was weak in all the cells $\left(2^{-\Delta \mathrm{Ct}}=0.0006 \pm 0.0002\right)$, while collagen II was only present in 5 of the 8 hTLC cultures at a negligible level $\left(2^{-\Delta \mathrm{Ct}}=0.00001 \pm 0.00001\right)$.

\section{Platelet and growth factor quantification in the PRP}

The platelet concentrations showed no significant differences between the 4 investigated donor groups. The median of the platelets was $3.6 \times 10^{8}$ platelets $/ \mathrm{ml}$ for the younger male group and $4.5 \times 10^{8}$ platelets $/ \mathrm{ml}$ for the 3 other groups (Fig. 2a).

The growth factor quantification revealed no significant differences between the groups. IGF-1 had the highest concentrations in the PRP, followed by TGF- $\beta 1$ and PDGF-AB (Fig. 2b). Only 1 of the 24 PRPs contained a very low amount of BMP-7 $(0.0004 \mathrm{pg} / \mu \mathrm{g})$, while low levels of BMP-12 $(0.021 \mathrm{pg} / \mu \mathrm{gg})$ were detected in 1 other sample. The PDGF-AB concentrations

Table 1 Primer

\begin{tabular}{llll}
\hline $\begin{array}{l}\text { Target } \\
\text { gene }\end{array}$ & $\begin{array}{l}\text { Accession } \\
\text { number }\end{array}$ & $\begin{array}{l}\text { Product } \\
\text { size }[\mathrm{bp}]\end{array}$ & Primer sequence [5' - 3'] \\
\hline GAPDH & NM_002046 & 115 & $\begin{array}{l}\text { Forward: CCACTCCTCCACCTITGACG } \\
\text { Reverse: CATGAGGTCACCACCCTGT }\end{array}$ \\
Col-I & NM_000088 & 197 & $\begin{array}{l}\text { Forward: TGACCTCAAGATGTGCCACT } \\
\text { Reverse: ACCAGACATGCCTCTTGTCC }\end{array}$ \\
Col-III & NM_000090 & 199 & $\begin{array}{l}\text { Forward: GCTGGCATCAAAGGACATCG } \\
\text { Reverse: TGTTACCTCGAGCCCTGGT }\end{array}$ \\
Osteocalcin & NM_199173 & 209 & $\begin{array}{l}\text { Forward: CCCAGGCGCTACCTGTATCAA } \\
\text { Reverse: CTGGAGAGAGCAGAACTGG }\end{array}$ \\
\hline
\end{tabular}

significantly correlated with the platelet concentrations in the PRP $\left(r_{s}=0.469, p=0.037\right)$. The correlation between the TGF- $\beta 1$ and PDGF-AB concentrations was also significant $\left(\mathrm{r}_{\mathrm{s}}=0.877, p<0.001\right)$.

\section{Cell proliferation}

Cell proliferation was significantly increased in all the groups by stimulation with PRP compared to the positive (10\% FCS, $p \leq 0.048)$ and negative controls (2\% FCS, $p \leq 0.0001)$. Significantly increased cell proliferation was further observed for all the hTLCs of the positive compared to the negative control group $(p \leq 0.0001)$. Additionally, in the group with the males over 65 years of age, the PRP showed significantly reduced stimulation potential compared to the group with the males under 65 years of age $(p=0.009)$. The cell proliferation did not correlate with the platelet or the growth factor concentrations of the PRP (Fig. 3).

\section{Collagen I protein synthesis}

In all the analyzed groups, the absolute collagen I synthesis (without normalization to cell proliferation) was significantly increased in the PRP-stimulated cells and the positive control group (10\% FCS) compared to the negative control group ( $2 \% \mathrm{FCS}, p \leq 0.005$ ), but not between the positive control (10\% $\mathrm{FCS})$ and the PRP-treated cells. In addition, the PRP stimulation of the female donors $(>/<65$ years) resulted in a significantly higher increase in total collagen I synthesis compared to the respective male groups ( $<65$ years: $p=0.043,>65$ years: $p=0.002$ ) (Fig. 4a).

With normalization to cell proliferation, the relative collagen I synthesis was significantly reduced in the PRP-treated cells compared to the hTLCs of the positive $(10 \% \mathrm{FCS}, p \leq 0.01)$ and negative controls ( $2 \% \mathrm{FCS}$, $p \leq 0.0001$ ), as well as in the positive compared to the negative control $(p \leq 0.03)$. In the younger male group, the PRP stimulation led to significantly reduced collagen I synthesis compared to the older male $(p=0.003)$ and the younger female groups $(p=0.006)$ (Fig. $4 \mathrm{~b})$. Correlation analysis revealed a significant negative correlation between the PDGF-AB concentration and the relative collagen I synthesis $\left(r_{s}=-0.502, p=0.017\right)$.

\section{Gene expression}

Collagen I expression was significantly reduced after PRP treatment compared to the positive control in the younger male $(p=0.016)$ and older female $(\mathrm{p}=0.016)$ groups. Additionally, in the hTLCs of the female donors over the age of 65 , the collagen I expression in the positive control was significantly increased compared to the negative control $(p=0.005)$. In the younger female group, the collagen I expression was significantly reduced compared to the negative $(\mathrm{p}=0.005)$ and positive 
Table 2 ELISA Kits

\begin{tabular}{|c|c|c|c|}
\hline Analyte & ELISA Kit & Specifics & $\begin{array}{l}\text { Dilution } \\
\text { of PRP }\end{array}$ \\
\hline PDGF-AB & $\begin{array}{l}\text { Quantikine human } \\
\text { PDGF-AB } \\
\text { (R\&D systems) }\end{array}$ & & $1: 50$ \\
\hline |GF-| & $\begin{array}{l}\text { Quantikine human } \\
\text { IGF-I (R\&D systems) }\end{array}$ & $\begin{array}{l}\text { PRP needs pre-treatment } \\
\text { (solution provided } \\
\text { in the kit) }\end{array}$ & $\begin{array}{l}\text { 1:100 (including } \\
\text { pre-treatment) }\end{array}$ \\
\hline TGF- $\beta 1$ & $\begin{array}{l}\text { Quantikine human } \\
\text { TGF- } \beta 1 \\
\text { (R\&D systems) }\end{array}$ & $\begin{array}{l}\text { PRP needs activation with } 1 \\
\mathrm{~N} \mathrm{HCL} \text {, neutralization with } \\
1.2 \mathrm{~N} \mathrm{NaOH} / 0.5 \mathrm{M} \mathrm{HEPES})\end{array}$ & $\begin{array}{l}\text { 1:40 (including } \\
\text { activation) }\end{array}$ \\
\hline BMP-7 & $\begin{array}{l}\text { DuoSet human } \\
\text { BMP-7 } \\
\text { (R\&D systems) }\end{array}$ & & Undiluted \\
\hline $\begin{array}{l}\text { BMP-12 } \\
\text { (GDF-7) }\end{array}$ & $\begin{array}{l}\text { Human GDF-7 } \\
\text { ELISA Kit } \\
\text { (Blue Gene) }\end{array}$ & Competitive ELISA & $1: 2$ \\
\hline
\end{tabular}

controls $(p=0.009)$. No significant differences were detected in the older male group (Fig. 5a).

The collagen III expression showed significant elevation in the male group under the age of 65 compared to the negative $(p=0.002)$ and positive controls $(p=0.004)$. In the hTLCs of the female donors under 65 , the collagen III expression was induced in the PRP-treated cells compared to the positive control $(p=0.016)$, but it was significantly lower in the positive compared to the negative control $(p=0.005)$. In the cells of the older female donors, the collagen III expression was significantly increased in the PRP compared to the negative control $(p=0.007)$. No significant differences were observed for the older male group (Fig. 5b).

The osteocalcin expression was unaffected by the PRP or FCS treatment of the hTLCs (Fig. 5c).

No correlations were detected between the growth factor content in the PRP and the gene expression of the hTLCs.

\section{Discussion}

The current study for the first time describes the stimulating effects of autologous PRP on the same patients tenocytes of the human rotator cuff. We hereby assessed parameters such as tenocyte proliferation as well as expression and synthesis of extracellular matrix components, particularly collagen I and III. were significantly improved (without normalization to cell proliferation). As discussed later, this finding is in general accordance with other in vitro studies in the field that describe enhanced cell proliferation, tendon matrix synthesis, and gene expression under allogenic PRP of different configurations $[13,17,22,24,46]$.

The effect of PRP was investigated in hTLCs of female and male subgroups of patients who were under and above 65 years of age. Some clinical cohort studies had suggested that a higher patient age is related to unsuccessful RC repair, but no consistent age limits have been defined [3, 11, 14, 19, 35, 37, 53, 61]. Furthermore, a sex-related effect had been advocated by some authors [10, 19], while others could not confirm this observation [35, 38, 54, 61].

If patient age or sex was a critical issue, standard RC repairs would be unsatisfactory in those beyond certain limits, possibly demanding additional biological RC repair augmentation, for example with growth factors obtained with PRP [1, 23, 24, 31, 40, 45, 49].

If, for instance, elderly female patients required additional biologic augmentation, autologous PRP might be one possible approach, as suggested in the present study. Recent in vitro studies have described age-related differences in biologic RC cell potential among male and female patients, with a weaker potential in those above the age of 65 [27, 28]. Tenocyte-like cells can be stimulated with growth factors such as BMP- 2 and -7 by means of
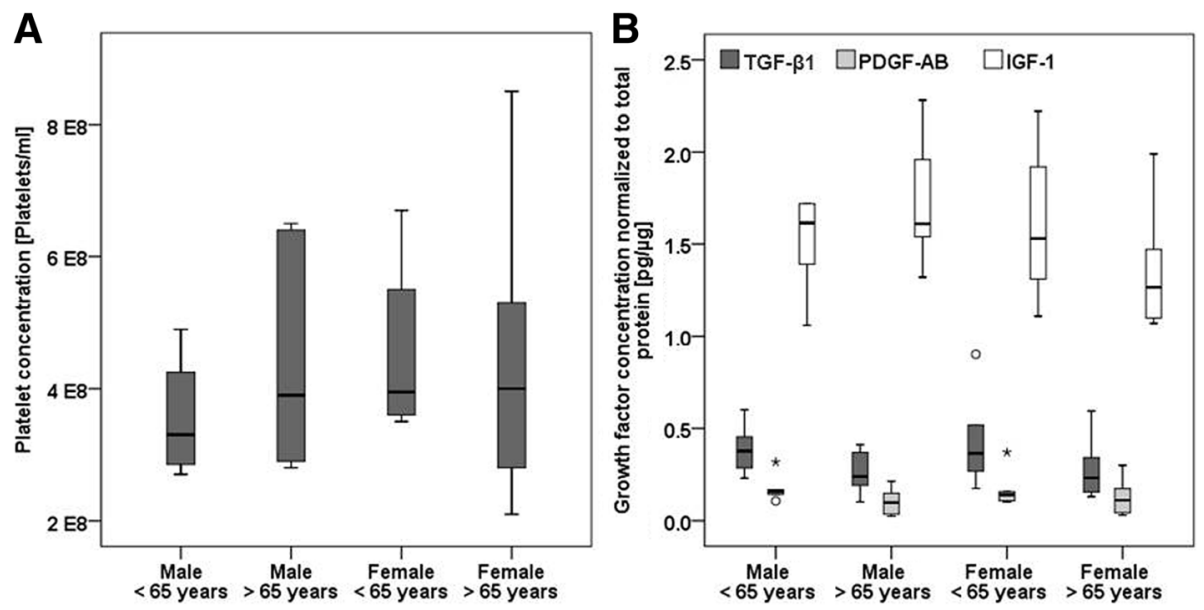

Fig. 2 a Platelet and $\mathbf{b}$ growth factor concentrations in the PRP of the four investigated donor groups. No significant differences were found between the groups. Stars and circles mark the outliers 


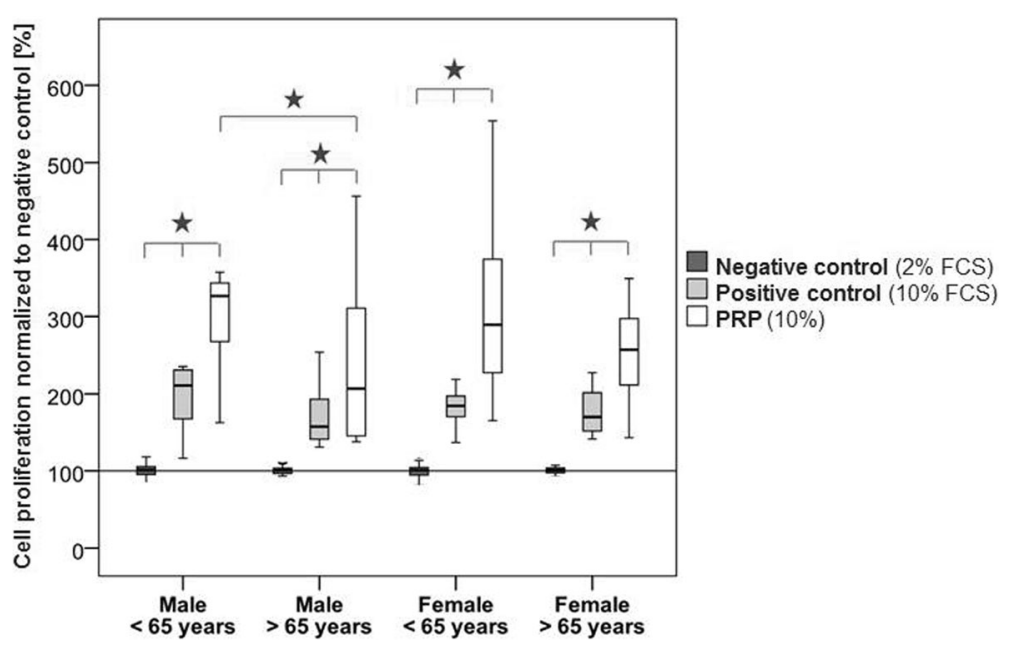

Fig. 3 Cell proliferation after PRP stimulation in the four investigated donor groups normalized to the negative control. The PRP led to significantly increased cell proliferation compared to the positive and negative controls. Additionally, the positive control showed an elevated cell proliferation compared to the negative control. Cell proliferation in the PRP-treated cells was significantly reduced in the older compared to the younger male groupStars indicate significant differences between the groups $(p \leq 0.05)$

increased cell activity and collagen I synthesis in elderly male and female patients [27, 28]. As discussed later, BMP-7 could not be quantified in the PRP preparation approach currently employed. However, the blend of other autologous growth factors herein demonstrated stimulating effects, suggesting that PRP might have potential when it comes to augmentation, particularly in this RC repair patient cohort with a possible biological demand.
Several methodological features of the present research differ from existing in vitro studies on PRP and tendon cells and provide new insights. A major strength of this study is that it is the first approach to investigate the effect of PRP on supraspinatus tenocytes of the same patient, representing the clinical and intraoperative application of autologous PRP. This approach requires a more elaborate protocol to allow for intraoperative tendon harvest, subsequent cell isolation, and
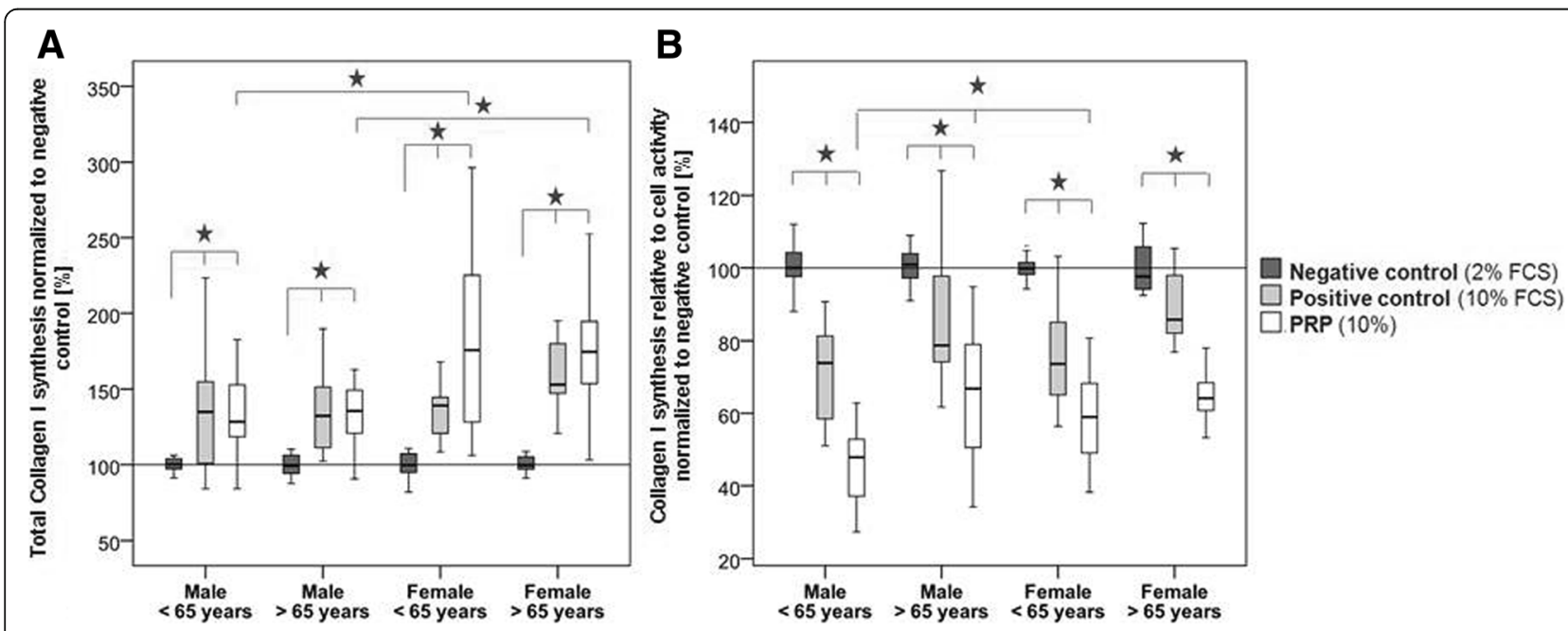

Fig. 4 Total and relative collagen I synthesis normalized to the negative control. a The total collagen I synthesis was significantly increased in the PRP group and the positive control compared to the negative control. PRP treatment led to significantly elevated effects in the hTLCs of the older compared to the younger donors. $\mathbf{b}$ Collagen I synthesis relative to cell proliferation was significantly reduced in the PRP group compared to the positive and negative controls, as well as in the positive compared to the negative control. In the younger male donors, the relative collagen I synthesis was significantly reduced compared to the older male and younger female groups. Stars indicate significant differences at $p \leq 0.05$ between groups 

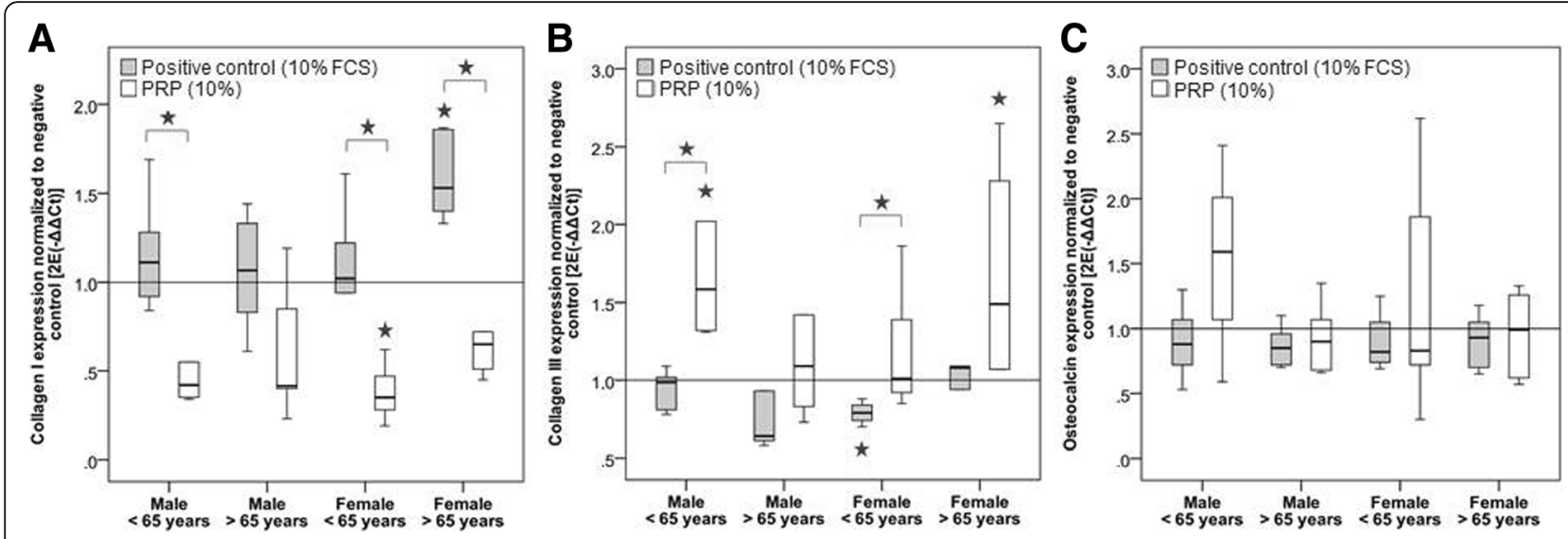

Fig. 5 Relative gene expression measured by qRT-PCR and normalized to GAPDH and the negative control (line). Stars above brackets mark significant differences between these groups at $p \leq 0.05$. Stars alone mark significant differences to the negative control. a Collagen I expression was significantly reduced in all the investigated groups except the older male group compared to the positive control. $\mathbf{b}$ The expression of collagen III was significantly increased in the PRP-treated cells compared to the positive and/or negative control in all the groups except the older males. c No significant differences were demonstrated for the osteocalcin expression

timed cell cultivation prior to scheduling patients for blood samples, PRP preparation, and application.

Furthermore, the present study features the largest number of enrolled donor patients of either sex or age group, and thus represents the typical patient cohort undergoing RC repair. Other studies in the field have assessed only monadic numbers of donors with allogenic PRP preparations [13, 17, 22, 24, 46]. Cross et al. involved $n=20 \mathrm{RC}$ samples, but these received allogenic PRP preparations and had irreparable and long-standing RC tears (cuff tear arthropathy), which does not represent the patient cohort undergoing RC repair (in contrast to the donors in the present study) [12].

Another strength of this study is that tissue samples were obtained from torn rotator cuffs at the site of repair (in contrast to intact rotator cuff material [55]). This may provide more specific insight into the biological effect of PRP on the targeted type of rotator cuff tissues when compared to tendon cultures with other origins [12]. In this regard, de Mos et al. suggested that tenocytes from degenerated and torn RCs might respond differently to PRP than the intact hamstring tendon cells of the young individuals investigated in their study [13]. However, their observation of increased tenocyte proliferation is in accordance with our present findings. The authors furthermore suggested a possible decrease of collagen synthesis per cell which is confirmed in our results, where total collagen I protein synthesis was increased but relative synthesis (normalized to cell proliferation) was significantly reduced. Could this be one reason why current clinical studies with this regard are rather disencouraging? With respect to the increased cell proliferation we believe it is more precise to differentiate between total and relative collagen I synthesis, even though it remains a gap of knowledge which of these parameters is the most relevant for successful clinical transfer. In addition, de Mos et al. observed reduced gene expression of collagen I, which again is in accordance with the present findings in torn $\mathrm{RC}$ tenocytes. The authors also described upregulation of matrix-degrading enzymes (matrix metalloproteinase - MMP - 1 and 3) in PRP-exposed cells, and concluded that PRP might accelerate the catabolic demarcation of traumatically injured tendon matrices [13]. We cannot compare these results to degenerative, torn RC tissue, as MMP expression was not assessed in the present study. However, Cross et al. described reduced matrix degradation and inflammation cytokines in elderly, torn RC-tenocytes treated with the same leukocyte-poor PRP preparation set as in the present study (Arthrex $\mathrm{ACP}^{\circ}$ ).

In the current research, tendon cell characterization was conducted prior to studying the cell response. This should be considered important enough to exclude the analysis of unspecific fibroblast cultures [41], and was conducted by some authors assessing the effect of PRP on tendon cells after characterization for tendon-specific markers such as scleraxis or decorin [17, 24]. In rodent tendon stem cells, PRP induced tenocyte differentiation, while adipocyte, chondrocyte, and osteocyte lineages were suppressed [8]. This reflects the present findings in human tenocytes, namely that osteocalcin expression was unaffected by PRP application.

A weakness of the present study is that no different PRP dosages were assessed, as other authors in this field have described previously $[13,17,46]$. The dose-dependent response of tenocytes to applied growth factors has, however, been reported elsewhere [40,62]. 
Growth factors (such as BMP 2 and 7) have been found to stimulate human RC cells, but dosages beyond the ideal concentration reduced tendon cell proliferation and collagen production [40]. Sadoghi et al. found a significant effect of PRP on RC fibroblast proliferation in vitro, with an optimal benefit using a onefold or 5 -fold, but not 10-fold, PRP concentration [46]. Further studies have demonstrated that increasing platelet concentrations in PRP applications beyond an ideal level does not result in further anabolic upregulation (in ani$\mathrm{mal} /$ human tenocytes) [4, 17]. In contrast, (too) high concentrations have been shown to have inhibitory effects on proliferation and collagen production, while MMP production increased, which is possibly counterproductive when it comes to tendon bone regeneration $[4,17]$. It has been suggested that an over-stimulation of cells might result in adverse effects, such as poorly-differentiated scar tissue at the insertion site [6, 57]. Accordingly, the chosen PRP concentration in the present study may not have been optimal, as the relative collagen I synthesis was significantly reduced (when normalized to cell proliferation). Moreover, an increase in PDGF-AB concentration in the PRP was found to correlate with a reduced relative collagen I synthesis, indicating that lower PRP concentrations could be beneficial. However, even if the optimal PRP dosage in vitro is known, it nevertheless remains challenging to transfer this knowledge to the clinical setting. It is technically difficult to influence or optimize growth factor dosages in the final PRP product intraoperatively, as this correlates to plasma viscosity and concentration at the time of venous blood sampling.

As the stimulation of osteoblasts is helpful for re-establishing the tendon-bone footprint following $\mathrm{RC}$ repair, growth factors with bone and tendon-stimulating potential (such as BMP-7 [26, 27, 40, 56, 62] and - 12 [29, $49,59])$ might be beneficial at the repair site. Accordingly, the current study aimed to quantify the amount of these factors to gain further insight, but the quantities were below the technical detection limit except for 1 donor with a low but detectable level of BMP-7 and 1 donor with a low level of BMP-12. This could either be a technical issue related to insufficient sensitivity of the kits or the fact that these respective growth factors are indeed absent in the PRP preparations.

To date, more clinical and radiographic studies on PRP and RC repairs have been published than in vitro studies on the biological characteristics of available PRP products. Some studies suggested a pain reduction following PRP application [16, 44]. However, recent reviews and meta-analyses found no significant effects of PRP augmentation in the treatment of full thickness RC tear repairs by means of outcome scores or re-tear rates $[5,7,21,30,47,51,60,63]$.
The question arises as to why the majority of clinical and radiographic studies on PRP augmentation in RC repair do not confirm the promising in vitro studies that generally describe positive effects of PRP on RC tendon cells.

One issue is the growth factor dose-dependent response of tendon cells and the difficulty of affecting their concentration in clinical practice, as discussed above. Yet other factors may also account for this controversy. Any analysis of the literature is complicated by the lack of standardization of study protocols, PRP preparation techniques, and the respective outcome measures in clinical studies [51].

There is a broad range of PRP preparations distributed in the market and employed in several studies. Yet it is not just the content and concentrations of platelets, white blood cells, and growth factors that differ significantly between different products and methods (1- versus double spin process), but also intra-individual variations of PRP products $[12,32,34]$. This variation may contribute to inhomogenous results, as may further variables in clinical studies such as the application time (intraoperatively/days later) and site (underneath the reconstructed tendon-bone unit or over the top) [60]. Finally, the actual surgical reconstruction technique (single vs. double row repair) and postoperative rehab protocols may further account for differences and hinder comparability.

These variables might contribute to the understanding of heterogeneous results in clinical and radiographic studies and the demand for further translational studies, while PRP generally shows effects on human RC tendon cells in vitro.

\section{Conclusions}

In this study, autologous PRP was a source of growth factors such as IGF-1, TGF- $\beta$, and PDGF-AB, but no detectable amounts of BMP-7 and -12 were found. PRP had an anabolic effect on the human rotator cuff tenocytes of the same individual in vitro by means of increased cell proliferation. On the other hand, and absolute, but nor relative collagen I synthesis and gene expression was significantly reduced and might explain limited clinical results of PRP applications. These results encourage further studies on clinical outcomes with more comparable standards in terms of preparation and application methods.

\section{Abbreviations}

BMP: Bone morphogenetic protein; COL: Collagen; hTLC: Human tenocytelike cells; IGF-1: Insulin-like growth factor; PDGF: Platelet-derived growth factor; PRP : Platelet rich plasma; RC: Rotator cuff; SSP: Supraspinatus; TGF$\beta$ : Transforming growth factor

\section{Acknowledgements}

The authors thank the funding sources named above to enable the present study. 


\section{Funding}

Financial support was provided by two non-profit organizations (the German and the European Society for Shoulder and Elbow Surgery: research grants) and by Arthrex $\mathrm{GmbH}$, Germany, by means of lab materials. None of the funding sources was involved with the design of the study, nor with collection, analysis, and interpretation of data, nor in writing the manuscript. The authors furthermore acknowledge support from the German Research Foundation (DFG) and the Open Access Publication Fund of Charite - Universitätsmedizin Berlin.

\section{Availability of data and materials}

All data are available by mail request via the author FKS (franka.klatte@charite.de).

\section{Authors' contributions}

SP developed the study design, analyzed data and wrote the manuscript. FK carried out the lab procedures, analyzed data and was a major contributor in writing the manuscript. KS carried out study coordination, assisted in lab procedures and statistical analysis. MS conceived of the study and participated in its design and coordination. BW conceived of the study, developed its design and critically revised the manuscript. All authors declare that this manuscript is original, has not been published before and is not currently being considered for publication elsewhere. It has been read and approved by all authors and each of the authors is convinced that the manuscript represents honest work

\section{Ethics approval and consent to participate}

Institutional review board approval was obtained from the Ethics committee at Charité Universitaetsmedizin Berlin, Germany (ref. nr. EA1/307/11). All participating patients written informed consent was obtained. Participation in the study did not affect the treatment algorithm.

\section{Consent for publication}

Not applicable. All patient data were anonymized.

\section{Competing interests}

All authors confirm that there are no conflicts of interest associated with this publication and there has been no financial support for this work that could have influenced its results.

\section{Publisher's Note}

Springer Nature remains neutral with regard to jurisdictional claims in published maps and institutional affiliations.

\section{Author details}

1 Julius Wolff Institut, Center for Musculoskeletal Surgery,

Charité-Universitaetsmedizin, Augustenburger Platz 1, 13353 Berlin, Germany. ${ }^{2}$ Berlin-Brandenburg Center for Regenerative Therapies,

Charité-Universitaetsmedizin, Berlin, Germany. ${ }^{3}$ Department of Experimental Traumatology, Universitaetsklinikum Jena, Jena, Germany.

Received: 14 June 2018 Accepted: 14 November 2018

\section{Published online: 30 November 2018}

\section{References}

1. Barber FA. Platelet-rich plasma for rotator cuff repair. Sports Med Arthrosc. 2013;21:199-205.

2. Bayne $\mathrm{O}$, Bateman J. Long term results of surgical repair of full thickness rotator cuff tears. In: Bateman J, Welsh R, editors. Surgery of the shoulder. Philadelphia: The C.V. Mosby Company, Inc; 1984.

3. Boileau P, Brassart N, Watkinson DJ, Carles M, Hatzidakis AM, Krishnan SG. Arthroscopic repair of full-thickness tears of the supraspinatus: does the tendon really heal? J Bone Joint Surg Am. 2005;87:1229-40.

4. Boswell SG, Schnabel LV, Mohammed HO, Sundman EA, Minas T, Fortier LA. Increasing platelet concentrations in leukocyte-reduced platelet-rich plasma decrease collagen gene synthesis in tendons. Am J Sports Med. 2014;42:42-9.

5. Cai YZ, Zhang C, Lin XJ. Efficacy of platelet-rich plasma in arthroscopic repair of full-thickness rotator cuff tears: a meta-analysis. J Shoulder Elb Surg. 2015;24:1852-9.
6. Cenni E, Avnet S, Fotia C, Salerno M, Baldini N. Platelet-rich plasma impairs osteoclast generation from human precursors of peripheral blood. J Orthop Res. 2010;28:792-7.

7. Chahal J, Van Thiel GS, Mall N, Heard W, Bach BR, Cole BJ, Nicholson GP Verma NN, Whelan DB, Romeo AA. The role of platelet-rich plasma in arthroscopic rotator cuff repair: a systematic review with quantitative synthesis. Arthroscopy. 2012;28:1718-27.

8. Chen L, Dong SW, Tao X, Liu JP, Tang KL, Xu JZ. Autologous platelet-rich clot releasate stimulates proliferation and inhibits differentiation of adult rat tendon stem cells towards nontenocyte lineages. J Int Med Res. 2012;40: 1399-409.

9. Chen M, Xu W, Dong Q, Huang Q, Xie Z, Mao Y. Outcomes of single-row versus double-row arthroscopic rotator cuff repair: a systematic review and meta-analysis of current evidence. Arthroscopy. 2013;29:1437-49.

10. Chung SW, Oh JH, Gong HS, Kim JY, Kim SH. Factors affecting rotator cuff healing after arthroscopic repair: osteoporosis as one of the independent risk factors. Am J Sports Med. 2011;39:2099-107.

11. Clayton RA, Court-Brown CM. The epidemiology of musculoskeletal tendinous and ligamentous injuries. Injury. 2008;39:1338-44.

12. Cross JA, Cole BJ, Spatny KP, Sundman E, Romeo AA, Nicholson GP, Wagner B, Fortier LA. Leukocyte-reduced platelet-rich plasma normalizes matrix metabolism in torn human rotator cuff tendons. Am J Sports Med. 2015;43:2898-906.

13. de Mos $M$, van der Windt $A E$, Jahr $H$, van Schie $H T$, Weinans $H$, Verhaar JA, van Osch GJ. Can platelet-rich plasma enhance tendon repair?A cell culture study. Am J Sports Med. 2008:36:1171-8.

14. DeFranco MJ, Bershadsky B, Ciccone J, Yum JK, lannotti JP. Functional outcome of arthroscopic rotator cuff repairs: a correlation of anatomic and clinical results. J Shoulder Elb Surg. 2007;16:759-65.

15. DeHaan AM, Axelrad TW, Kaye E, Silvestri L, Puskas B, Foster TE. Does double-row rotator cuff repair improve functional outcome of patients compared with single-row technique? A systematic review. Am J Sports Med. 2012:40:1176-85.

16. Flury M, Rickenbacher D, Schwyzer HK, Jung C, Schneider MM, Stahnke K, Goldhahn J, Audige L. Does pure platelet-rich plasma affect postoperative clinical outcomes after arthroscopic rotator cuff repair? A Randomized Controlled Trial. Am J Sports Med. 2016:44:2136-46.

17. Giusti I, D'Ascenzo S, Manco A, Di Stefano G, Di Francesco M, Rughetti A, Dal Mas A, Properzi G, Calvisi V, Dolo V. Platelet concentration in platelet-rich plasma affects tenocyte behavior in vitro. Biomed Res Int. 2014;2014:630870.

18. Goutallier D, Postel JM, Bernageau J, Lavau L, Voisin MC. Fatty muscle degeneration in cuff ruptures. Pre- and postoperative evaluation by CT scan. Clin Orthop Relat Res. 1994;(304):78-83.

19. Grasso A, Milano G, Salvatore M, Falcone G, Deriu L, Fabbriciani C. Singlerow versus double-row arthroscopic rotator cuff repair: a prospective randomized clinical study. Arthroscopy. 2009:25:4-12.

20. Gulotta LV, Rodeo SA. Growth factors for rotator cuff repair. Clin Sports Med. 2009:28:13-23.

21. Gwinner C, Gerhardt C, Haneveld H, Scheibel M. Two-staged application of PRP in arthroscopic rotator cuff repair: a matched-pair analysis. Arch Orthop Trauma Surg. 2016;136:1165-71.

22. Hilber F, Loibl M, Lang S, Kerschbaum M, Brockhoff G, Angele P, Zellner J, Schmitz P, Nerlich M, Worlicek M. Leukocyte-reduced platelet-rich plasma increases proliferation of tenocytes treated with prednisolone: a cell cycle analysis. Arch Orthop Trauma Surg. 2017;137:1417-22.

23. Isaac C, Gharaibeh B, Witt M, Wright VJ, Huard J. Biologic approaches to enhance rotator cuff healing after injury. J Shoulder Elb Surg. 2012; 21:181-90.

24. Jo CH, Kim JE, Yoon KS, Shin S. Platelet-rich plasma stimulates cell proliferation and enhances matrix gene expression and synthesis in tenocytes from human rotator cuff tendons with degenerative tears. Am J Sports Med. 2012:40:1035-45.

25. Kevy SV, Jacobson MS. Comparison of methods for point of care preparation of autologous platelet gel. J Extra Corpor Technol. 2004;36:28-35.

26. Klatte-Schulz F, Gerhardt C, Scheibel M, Wildemann B, Pauly S. Relationship between muscle fatty infiltration and the biological characteristics and stimulation potential of tenocytes from rotator cuff tears. J Orthop Res. 2014:32:129-37.

27. Klatte-Schulz F, Pauly S, Scheibel M, Greiner S, Gerhardt C, Hartwig J, Schmidmaier G, Wildemann B. Characteristics and stimulation potential with BMP-2 and BMP-7 of tenocyte-like cells isolated from the rotator cuff of female donors. PLoS One. 2013;8:e67209. 
28. Klatte-Schulz F, Pauly S, Scheibel M, Greiner S, Gerhardt C, Schmidmaier G, Wildemann B. Influence of age on the cell biological characteristics and the stimulation potential of male human tenocyte-like cells. Eur Cell Mater. 2012:24:74-89.

29. Kovacevic D, Rodeo SA. Biological augmentation of rotator cuff tendon repair. Clin Orthop Relat Res. 2008;466:622-33.

30. Li X, Xu CP, Hou YL, Song JQ, Cui Z, Yu B. Are Platelet Concentrates An Ideal Biomaterial for Arthroscopic Rotator Cuff Repair? A Meta-Analysis of Randomized Controlled Trials. Arthroscopy. 2014;30(11):1483-90.

31. Lorbach O, Baums MH, Kostuj T, Pauly S, Scheibel M, Carr A, Zargar N, Saccomanno MF, Milano G. Advances in biology and mechanics of rotator cuff repair. Knee Surg Sports Traumatol Arthrosc. 2015;23:530-41.

32. Magalon J, Bausset O, Serratrice N, Giraudo L, Aboudou H, Veran J, Magalon G, Dignat-Georges F, Sabatier F. Characterization and comparison of 5 platelet-rich plasma preparations in a single-donor model. Arthroscopy. 2014;30:629-38.

33. Marx RE. Platelet-rich plasma: evidence to support its use. J Oral Maxillofac Surg. 2004;62:489-96.

34. Mazzocca AD, McCarthy MB, Chowaniec DM, Cote MP, Romeo AA, Bradley JP, Arciero RA, Beitzel K. Platelet-rich plasma differs according to preparation method and human variability. J Bone Joint Surg Am. 2012;94:308-16.

35. Milgrom C, Schaffler M, Gilbert S, van Holsbeeck M. Rotator-cuff changes in asymptomatic adults. The effect of age, hand dominance and gender. J Bone Joint Surg Br. 1995;77:296-8.

36. Molloy T, Wang Y, Murrell G. The roles of growth factors in tendon and ligament healing. Sports Med. 2003;33:381-94.

37. Ogata S, Uhthoff HK. Acromial enthesopathy and rotator cuff tear. A radiologic and histologic postmortem investigation of the coracoacromial arch. Clin Orthop Relat Res. 1990;(254):39-48.

38. Osti L, Papalia R, Del Buono A, Denaro V, Maffulli N. Comparison of arthroscopic rotator cuff repair in healthy patients over and under 65 years of age. Knee Surg Sports Traumatol Arthrosc. 2010;18:1700-6.

39. Pauly S, Gerhardt C, Chen J, Scheibel M. Single versus double-row repair of the rotator cuff: does double-row repair with improved anatomical and biomechanical characteristics lead to better clinical outcome? Knee Surg Sports Traumatol Arthrosc. 2010a;18:1718-29.

40. Pauly S, Klatte F, Strobel C, Schmidmaier G, Greiner S, Scheibel M, Wildemann B. BMP-2 and BMP-7 affect human rotator cuff tendon cells in vitro. J Shoulder Elb Surg. 2011:21:464-73.

41. Pauly S, Klatte F, Strobel C, Schmidmaier G, Greiner S, Scheibel M, Wildemann B. Characterization of tendon cell cultures of the human rotator cuff. Eur Cell Mater. 2010b;20:84-97.

42. Perser K, Godfrey D, Bisson L. Meta-analysis of clinical and radiographic outcomes after arthroscopic single-row versus double-row rotator cuff repair. Sports Health. 2011;3:268-74.

43. Prasathaporn N, Kuptniratsaikul S, Kongrukgreatiyos K. Single-row repair versus double-row repair of full-thickness rotator cuff tears. Arthroscopy. 2011;27:978-85.

44. Randelli P, Arrigoni P, Ragone V, Aliprandi A, Cabitza P. Platelet rich plasma in arthroscopic rotator cuff repair: a prospective RCT study, 2-year follow-up. J Shoulder Elb Surg. 2011;20:518-28.

45. Rodeo SA. Biologic augmentation of rotator cuff tendon repair. J Shoulder Elb Surg. 2007;16:S191-7.

46. Sadoghi $P$, Lohberger $B$, Aigner B, Kaltenegger $H$, Friesenbichler J, Wolf $M$, Sununu T, Leithner A, Vavken P. Effect of platelet-rich plasma on the biologic activity of the human rotator-cuff fibroblasts: a controlled in vitro study. J Orthop Res. 2013:31:1249-53.

47. Saltzman BM, Jain A, Campbell KA, Mascarenhas R, Romeo AA, Verma NN, Cole BJ. Does the Use of Platelet-Rich Plasma at the Time of Surgery Improve Clinical Outcomes in Arthroscopic Rotator Cuff Repair When Compared With Control Cohorts? A Systematic Review of Meta-analyses. Arthroscopy. 2016;32(5):906-18. https://doi.org/10.1016/j.arthro.2015.10.007. Epub 2015 Dec 23

48. Saridakis $\mathrm{P}$, Jones $\mathrm{G}$. Outcomes of single-row and double-row arthroscopic rotator cuff repair: a systematic review. J Bone Joint Surg Am. 2010;92:732-42.

49. Seeherman HJ, Archambault JM, Rodeo SA, Turner AS, Zekas L, D'Augusta D, Li XJ, Smith E, Wozney JM. rhBMP-12 accelerates healing of rotator cuff repairs in a sheep model. J Bone Joint Surg Am. 2008;90:2206-19.

50. Sheibani-Rad S, Giveans MR, Arnoczky SP, Bedi A. Arthroscopic single-row versus double-row rotator cuff repair: a meta-analysis of the randomized clinical trials. Arthroscopy. 2013;29:343-8.
51. Sheth U, Simunovic N, Klein G, Fu F, Einhorn TA, Schemitsch E, Ayeni OR, Bhandari M. Efficacy of autologous platelet-rich plasma use for orthopaedic indications: a meta-analysis. J Bone Joint Surg Am. 2012;94:298-307.

52. Slater M, Patava J, Kingham K, Mason RS. Involvement of platelets in stimulating osteogenic activity. J Orthop Res. 1995;13:655-63.

53. Sorensen AK, Bak K, Krarup AL, Thune CH, Nygaard M, Jorgensen U, Sloth C, Torp-Pedersen S. Acute rotator cuff tear: do we miss the early diagnosis? A prospective study showing a high incidence of rotator cuff tears after shoulder trauma. J Shoulder Elb Surg. 2007;16:174-80.

54. Tashjian RZ, Hollins AM, Kim HM, Teefey SA, Middleton WD, Steger-May K, Galatz LM, Yamaguchi K. Factors affecting healing rates after arthroscopic double-row rotator cuff repair. Am J Sports Med. 2010;38:2435-42.

55. Tempfer H, Gehwolf R, Lehner C, Wagner A, Mtsariashvili M, Bauer HC, Resch $\mathrm{H}$, Tauber M. Effects of crystalline glucocorticoid triamcinolone acetonide on cultered human supraspinatus tendon cells. Acta Orthop. 2009:80(3):357-62. https://doi.org/10.3109/17453670902988360.

56. Tsai AD, Yeh LC, Lee JC. Effects of osteogenic protein-1 (OP-1, BMP-7) on gene expression in cultured medial collateral ligament cells. J Cell Biochem. 2003:90:777-91.

57. Vavken P, Sadoghi P, Murray MM. The effect of platelet concentrates on graft maturation and graft-bone interface healing in anterior cruciate ligament reconstruction in human patients: a systematic review of controlled trials. Arthroscopy. 2011;27:1573-83

58. Vavken $P$, Sadoghi $P$, Palmer M, Rosso C, Mueller AM, Szoelloesy G, Valderrabano V. Platelet-rich plasma reduces Retear rates after arthroscopic repair of small- and medium-sized rotator cuff tears but is not cost-effective. Am J Sports Med. 2015;43(12):3071-6.

59. Violini S, Ramelli P, Pisani LF, Gorni C, Mariani P. Horse bone marrow mesenchymal stem cells express embryo stem cell markers and show the ability for tenogenic differentiation by in vitro exposure to BMP-12. BMC Cell Biol. 2009;10:29.

60. Warth RJ, Dornan GJ, James EW, Horan MP, Millett PJ. Clinical and structural outcomes after arthroscopic repair of full-thickness rotator cuff tears with and without platelet-rich product supplementation: a meta-analysis and meta-regression. Arthroscopy. 2015;31:306-20.

61. Yamaguchi K, Ditsios K, Middleton WD, Hildebolt CF, Galatz LM, Teefey SA. The demographic and morphological features of rotator cuff disease a comparison of asymptomatic and symptomatic shoulders. J Bone Joint Surg Am. 2006:88:1699-704.

62. Yeh LC, Tsai AD, Lee JC. Bone morphogenetic protein-7 regulates differentially the mRNA expression of bone morphogenetic proteins and their receptors in rat achilles and patellar tendon cell cultures. J Cell Biochem. 2008;104:2107-22.

63. Zhang Q, Ge H, Zhou J, Cheng B. Are platelet-rich products necessary during the arthroscopic repair of full-thickness rotator cuff tears: a metaanalysis. PLoS One. 2013;8:e69731.

Ready to submit your research? Choose BMC and benefit from

- fast, convenient online submission

- thorough peer review by experienced researchers in your field

- rapid publication on acceptance

- support for research data, including large and complex data types

- gold Open Access which fosters wider collaboration and increased citations

- maximum visibility for your research: over $100 \mathrm{M}$ website views per year

At $\mathrm{BMC}$, research is always in progress.

Learn more biomedcentral.com/submission 